\title{
Esquistossomose: reprodução e expansão da endemia no Estado de Pernambuco no Brasil
}

\author{
Schistosomiasis: reproduction and expansion of the \\ endemic region in Brazil
}

\author{
Constança Simões Barbosa, Carlos Bernardo da Silva e Frederico Simões Barbosa \\ Departamento de Parasitologia do Centro de Pesquisas Aggeu Magalhães da Fundação Oswaldo \\ Cruz. Recife, PE - Brasil (C.S.B., F.S.B.), Fundação Nacional de Saúde. Recife, PE - Brasil (C.B.S.)
}

\begin{abstract}
Resumo
A esquistossomose continua a ser um problema de saúde pública no Nordeste do Brasil embora o emprego, em larga escala, da quimioterapia venha sendo apontado como um dos fatores responsáveis pela redução das formas graves. $\mathrm{O}$ Estado de Pernambuco vem apresentando taxas crescentes de infecção humana para esquistossomose com perfil epidemiológico de prevalências crônicas (até $80 \%$ ) na região rural e casos recentes de infecção aguda no litoral. Discute-se a reprodução e expansão da esquistossomose a partir de uma concepção estrutural e histórica de causas, onde se inserem fatores não só de ordem biológica mas também sociais, políticos e culturais que vêm contribuindo para a formação dos quadros endêmicos: a forma de ocupação e do uso da terra, desemprego, desnutrição, migração e outros. Questionam-se as crescentes dificuldades para o controle da doença e o papel da investigação epidemiológica na compreensão da essência social do processo saúde/doença.
\end{abstract}

Esquistossomose mansoni, epidemiologia.

\begin{abstract}
Schistosomiasis mansoni can be considered an important public health problem in Northeastern Brazil, in spite of the reduction in the prevalence of the hepatosplenic clinical forms which have been attributed to the large scale use of chemotherapy in this country. However, the rise in the prevalence rates and the spread of this endemic disease to new areas show that schistosomiasis is assuming its must cruel expression: less lethal but more greatly incapacitating in terms of irreversible physical and moral damage to human beings. The state of Pernambuco presents growing rates for schistosomiasis infection in humans. The epidemiological profile of this disease displays high and consistent prevalence rates (up to 80\%) in rural areas, and new cases of acute infection on the coast, where schistosomiasis has recently been introduced. The reproduction and expansion of this endemic disease can be better understood on the basic of a conception of structural and historical causation. The disease construction process should be reconstructed in the light of biological as well as the social, political and cultural factors which are jointly responsible for the present endemic situation. Within that frame work, the historical and socioeconomic features that interact with the parasite and give rise to the present
\end{abstract}

Correspondência para/Correspondence to: Constança Simões Barbosa - Departamento de Parasitologia do Centro de Pesquisa Aggeu Magalhães da Fundação Oswaldo Cruz. Campus da Universidade Federal de Pernambuco - 50670-420 Recife, PE - Brasil.

Recebido em 6.10.1995. Reapresentado em 19.4.1996. Aprovado em 17.5.1996. 
proportions of the schistosomiasis epidemic in Pernambuco are discussed. The mode of occupation and use of the land, unemployment, under-nutrition, migration, etc., raise the question of the growing difficulties confronting the control of the disease, both in rural areas where populations are extremely mobile as well as in the poorly organized urban population. Epidemiological investigation is fulfithing its role in its attenpts to understand the complex relationships of an intrinsecally social nature of the health/disease process between health problems and the quality of life for the purpose of producing consistent disease control models.

Schistosomiasis mansoni, epidemiology.

\section{INTRODUÇÃO}

A esquistossomose continua a ser um problema de saúde pública no Nordeste do Brasil. O emprego, em larga escala, da quimioterapia tem sido apontado como um dos fatores responsáveis pela redução das formas graves, letais, da doença $\left(\mathrm{WHO}^{24}\right.$, 1993). Paralelamente, as prevalências humanas e o estabelecimento de novos focos de transmissão ativa da parasitose continuam em expansão fazendo com que a endemia assuma uma expressão cada vez mais cruel: menos letal, porém largamente incapacitante, provocando danos físicos e morais irreversíveis nas populações afetadas.

O modelo de saúde pública vigente entra em crise diante do aumento das taxas de incidência e prevalência das endemias, recolocando a questão da validade desses modelos de explicação, métodos de investigação e procedimentos de intervenção sobre o processo de produção das doenças $\left(\right.$ Sabroza $\left.^{16}, 1991\right)$. Essa reprodução continuada da esquistossomose não pode ser explicada nem detida através de abordagem puramente biologicista, pois esta tem se mostrado insuficiente no entendimento e controle da doença, que deveria ser compreendida como um processo histórico, gerador de uma estrutura epidemiológica, onde estão contidos os vários níveis determinantes de sua atual ocorrência. Fatores biológicos, sociais, políticos e culturais vêm contribuindo para a formação de quadros endêmicos específicos.

Nas raízes históricas do desenvolvimento social e econômico do Brasil-colônia é que se encontra as condições objetivas para a endemização da esquistossomose. A concessão de vastas extensões de terra à nobreza feudal fez surgir a necessidade da mãode-obra escrava, importada da África juntamente com o parasito Schistosoma mansoni.

Essa forma originária de organização social para a produção rural, com sistema de latifúndios e ex- ploração da força humana de trabalho, vem se perpetuando até os dias atuais em algumas regiões brasileiras, sendo um modelo excludente e gerador de miséria e doenças.

Os programas de controle de endemias também fazem parte do cenário político como perpetuadores da situação endêmica. E isto porque estão condicionados a pressões externas de multinacionais que delimitam as medidas oficiais de intervenção ao tratamento quimioterápico dos indivíduos doentes e à aplicação sistemática de moluscicidas, ações essas, comprovadamente paliativas e repetitivas, além de tóxicas e poluidoras. Essas ações programáticas situamse nas bordas do processo, agindo exclusivamente sobre o efeito ou sobre as manisfestações físicas da doença, e nunca questionando suas causas históricas e estruturais.

O processo estrutural da produção da esquistossomose passa dentro de um espaço socialmente construído e se expressa nas condições de vida das populações. O modo como esse espaço é ocupado garante a reprodução da doença e a distribuição desigual dos diferentes grupos de risco. A forma de inserção no processo produtivo vai definir quais os subgrupos populacionais com maior ou menor risco de adquirir a esquistossomose e desenvolver diferentes gradações de morbidade. De acordo com Loureiro ${ }^{13}$ (1989), e com algumas considerações adicionais, o processo estrutural da doença pode ser analisado, didaticamente, em três níveis determinantes:

\section{- Bioecológico}

Condições ambientais que otimizam a reprodução dos vetores e propiciam a sobrevivência do parasito.

- Socioecológico

Características ambientais modificadas pela ocupação social do espaço (construção de açudes, sistemas de irrigação, práticas agrícolas, forma de eliminação dos dejetos, e outros). 


\section{- Sociocultural}

Esse nível de análise possui uma dimensão supraestrutural, uma vez que contém os determinantes macro (históricos, políticos), embora sua elaboração final se dê no nível particular, estrutural, relacionado com a representação social da doença, ou seja, a construção cultural, o conhecimento e percepções que os grupos sociais têm sobre a esquistossomose. Esse nível condicionante contribui para a conformação do comportamento dos indivíduos e grupos sociais, suas atitudes e práticas de risco.

A vantagem de se abordar essa estrutura epidemiológica desde a análise do espaço socialmente construído e ocupado pela doença, está em se captar o processo gerador dessa estrutura e não somente descrevê-la estaticamente $\left(\right.$ Silva $\left.^{20}, 1990\right)$.

O presente trabalho tem a intenção de contribuir para a compreensão do processo endêmico da esquistossomose em algumas localidades do Estado de Pernambuco e considerar as perspectivas de controle da endemia, em nível local, partindo de uma concepção estrutural e histórica de causas.

Para os estudos de evolução das prevalências para S. mansoni foram elegidas algumas localidades, em áreas endêmicas da zona da mata do Estado de Pernambuco, a partir do critério de disponibilidade de informação sobre a evolução da morbidade para esquistossomose, nessas comunidades. Nos municípios de São Lourenço da Mata, Vitória de Santo Antão e Tracunhaém foi possível levantar 12 localidades e no Município de Paudalho, 9 localidades, todas elas com características ecológicas e ambientais semelhantes, população agrícola e mesmo tipo de vetor. O Centro de Pesquisas Aggeu Magalhães vem trabalhando há alguns anos nessas localidades, e os dados de prevalências anteriores e de tratamento foram levantados: na Fundação Nacional de Saúde (Recife, PE), através de consultas em material das campanhas de esquistossomose, desde 1984; nos boletins epidemiológicos, de coproscopia e quimioterapia de inquéritos realizados em populações de área endêmica da zona da mata do Estado de Pernambuco.

Complementarmente foi realizado levantamento de bibliografia específica na área das ciências sociais, para subsidiar a construção teórica do processo endêmico da esquistossomose em Pernambuco, resgatando elementos históricos, econômicos, sociais e culturais de eventos relacionados à produção e expansão da endemia: migrações, formas de ocupação do espaço rural e de produção agrícola, entre outros.

\section{Situação da Esquistossomose em Pernambuco}

Dados do órgão oficial de controle da esquistossomose, FNS (Fundação Nacional de Saúde), indicam que Pernambuco está entre os Estados que exibem prevalência média mais elevada de pessoas infectadas pelo Schistosoma mansoni (Tabela). Ainda assim, este indicador de morbidade não retrata especificidades locais, como, por exemplo, populações humanas onde as prevalências chegam acima de $80 \%$. Os arquivos da FNS evidenciam a estabilidade da doença em certas localidades da zona canavieira, onde vários tratamentos na população infectada e aplicações de moluscicida não conseguiram reverter, a médio prazo, a prevalência da parasitose (Figuras 1 e 2).

Tabela - Prevalência média para a esquistossomose em alguns Estados do Nordeste.

\begin{tabular}{lcccc}
\hline $\begin{array}{l}\text { Unidades da } \\
\text { Federação }\end{array}$ & $\begin{array}{c}\text { Localidades } \\
\text { trabalhadas }\end{array}$ & $\begin{array}{c}\text { Exames } \\
\text { realizados }\end{array}$ & $\begin{array}{c}\text { Exames } \\
\text { positivos }\end{array}$ \\
\hline Paraíba & 135 & 32.768 & 3.617 & 11,0 \\
Pernambuco & 425 & 80.584 & 12.281 & 15,2 \\
Alagoas & 152 & 20.856 & 9.023 & 43,3 \\
Sergipe & 115 & 51.105 & 7.141 & 14,0 \\
\hline Fonte: Setor de Informática da Fundação Nacional de Saúde, Recife, \\
Pernambuco.
\end{tabular}

Em localidades onde vêm sendo desenvolvidos estudos experimentais de controle (Pieri, 1995)*, com intervenção sistemática de quimioterapia humana e aplicação de moluscicida, o resíduo de casos positivos tem sido suficiente para manter, em níveis baixos, as taxas endêmicas, que, cessada a intervenção, tenderão a voltar rapidamente aos níveis anteriores (Figura 3).

O último tratamento em massa do programa oficial de controle da esquistossomose, da FNS, foi em 1986. Após esta data, o efetivo humano e as viaturas da FNS, lotados no citado programa, foram desviados para as campanhas emergenciais da dengue e cólera, só voltando a atuar recentemente.

O Estado de Pernambuco, mesmo com todos os esforços da FNS, apresenta taxas de infecção humana crescentes, passando por situações de prevalências crônicas, na região da zona da mata, com até $80 \%$ de indivíduos parasitados em certas localidades (Barbosa e Silva ${ }^{3}$, 1992), ou ainda, casos de infecção aguda freqüentes, como os que vêm ocorrendo no litoral do Estado, em áreas urbanas, de recente introdução da doença (Gonçalves e col. $\left.{ }^{9}, 1992\right)$. Focos de es-

\footnotetext{
* Projeto em andamento por O. S. Pieri, sobre "Controle de caramujos conjugados com quimioterapia em área endêmica para esquistossomose na zona canavieira do Nordeste. Instituto Oswaldo Cruz e Centro de Pesquisas Aggeu Magalhães (FIOCRUZ), Recife, 1995.
} 


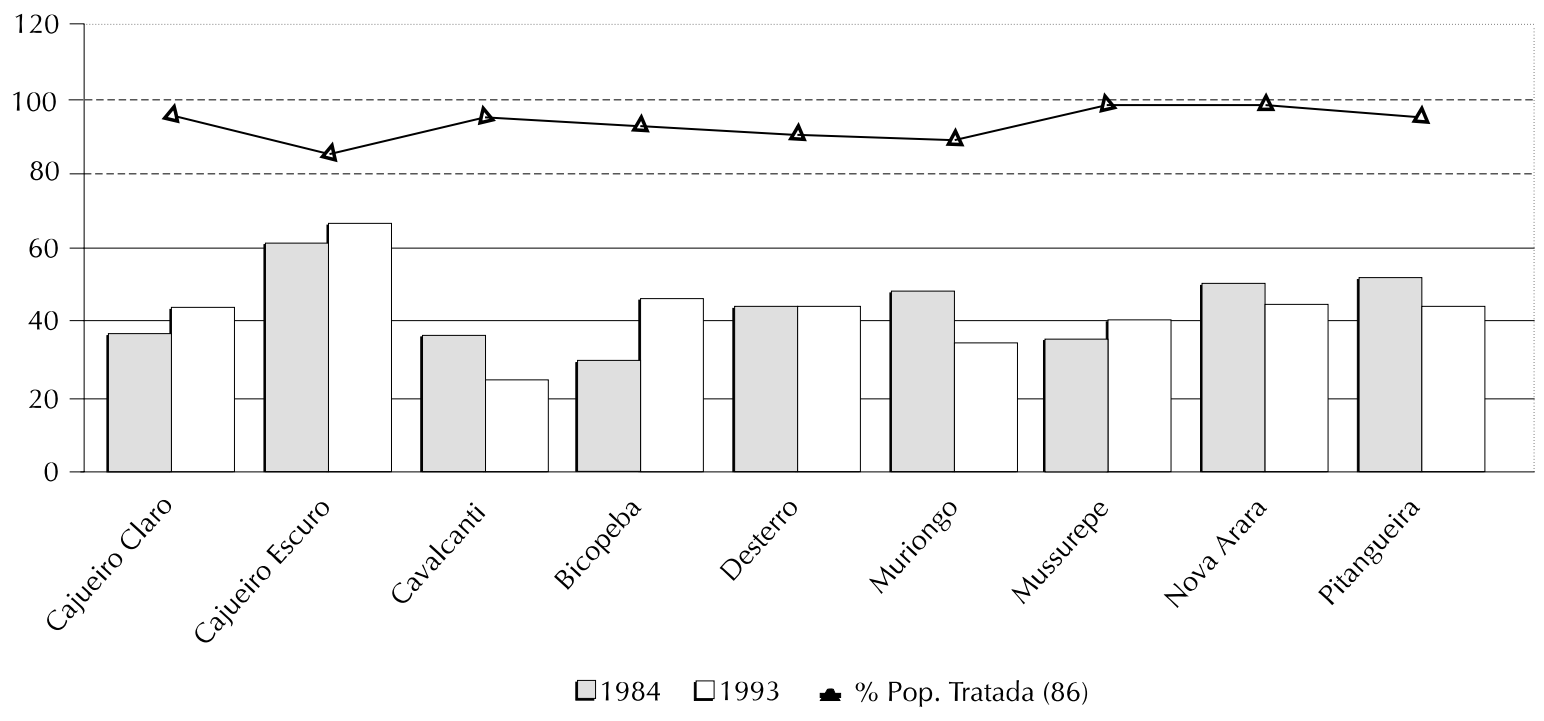

Figura 1 - Evolução das prevalências de esquistossomose, antes e após tratamento em massa, Município de Paudalho, PE. Fonte: Setor de Informática da Fundação Nacional de Saúde - Recife, Pernambuco.

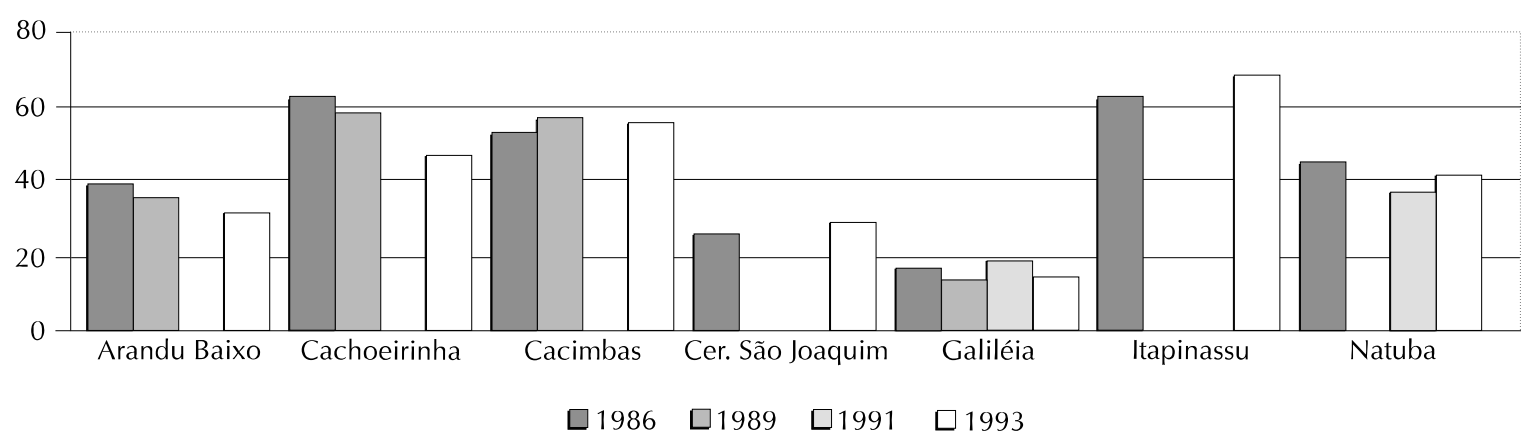

Figura 2 - Evolução das prevalências de esquistossomose, antes e após tratamento em massa, Municípios de Vitória de Santo Antão e Tracunhaém, PE.

Fonte: 1986 - Fundação Nacional de Saúde - Pernambuco.

1989, 1991 e 1993 - CPqAM / FIOCRUZ / Pernambuco.

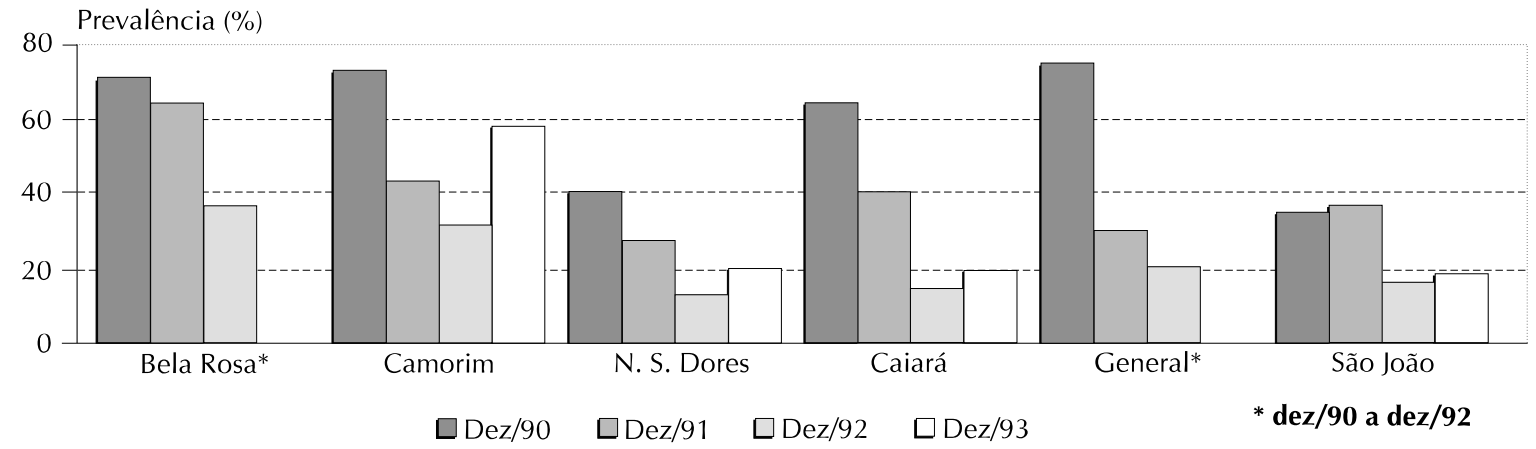

Figura 3 - Evolução das prevalências de esquistossomose, após tratamento quimioterápico seletivo anual, em localidades do Município de São Lourenço da Mata, PE.

Fonte: O. Pieri - Projeto em andamento - FIOCRUZ. 
quistossomose vêm surgindo no Grande Recife, em locais de povoamento intenso ou recente, como favelas e alojamentos para trabalhadores da construção civil (Samico $\left.{ }^{17}, 1989\right)$.

Os programas de controle da endemia, mesmo instrumentalizados com tecnologias eficazes, não têm conseguido eficiência nas suas ações porque são pensados a partir de uma lógica centralizada, intervencionista, medicalizada e padronizada para todas as áreas endêmicas.

As práticas operacionais desses programas têm se limitado à aplicação de moluscicida e ao tratamento quimioterápico das pessoas infectadas. Coutinho $^{8}$ (1981), avaliando o Programa PECE (Programa Especial de Controle da Esquitossomose) em relação ao controle da morbidade em Pernambuco, observa que:

- houve queda da intensidade da infecção e, conseqüentemente, inibição potencial na evolução para formas graves, devido não só ao PECE mas a tratamentos anteriores.

- nas localidades hiperendêmicas, as prevalências, após queda inicial, vêm retornando a níveis próximos dos anteriores à instalação do PECE.

\section{FATOS HISTÓRICOS}

Alguns fatos históricos e socioeconômicos, juntamente com os fatores de ordem biológica, foram responsáveis pela atual expressão da endemia em Pernambuco. Tradicionalmente, o cultivo da cana-deaçúcar em Pernambuco foi desenvolvido nos vales úmidos (irrigados por rios) e colinas suaves (encostas), que fisiograficamente constituem a zona da mata, originariamente ocupada por vegetação típica de floresta (Mata Atlântica) que se estendia perpendicularmente ao litoral, desde o Rio Grande do Norte até Sergipe. Posteriormente, as áreas de cultivo de cana foram estendidas até as formações de tabuleiros que assumem diferentes morfologias: matas, chãs, grotas, várzeas e cerrados. Essa formação (ou área cultivável) avança para o interior dos Estados penetrando até $40 \mathrm{~km}$ a oeste. A necessidade de expansão da área de cultivo da cana-de-açúcar deu-se em função da "modernização" do cultivo, ditada pela introdução das destilarias de álcool, sendo que esse avanço do latifúndio marcou também a expropriação dos pequenos produtores das terras que ocupavam (Heredia ${ }^{10}, 1989$ ).

A grande maioria dos trabalhadores da zona da mata canavieira vive exclusivamente da venda de sua força de trabalho (assalariado). Raros são os que têm controle de pequenas porções de terra (pequenos proprietários, arrendatários ou meeiros) e as cultivam visando ao auto abastecimento e à venda do excedente (Andrade², 1986).

O Programa Pró-Álcool, a crescente valorização das terras e a mecanização tecnológica recrudesceram a política de exploração latifundiária e mudaram as relações de trabalho agrícola: os usineiros ampliaram as áreas de plantação de cana, passando a proibir o plantio de culturas de subsistência e expulsando da terra a mão-de-obra excedente.

Atualmente, prática muito utilizada é o emprego de mão-de-obra diária, os bóias-frias, trazidos de outras localidades, que cortam a cana, recebem o pagamento do dia e são levados de volta a seus lugares de origem. Com isso, o proprietário de terras beneficiase duplamente: só emprega mão-de-obra aparentemente saudável, livra-se de encargos trabalhistas e de movimentos populares de reinvidicações pela posse da terra, garantindo a manutenção da estrutura fundiária no campo.

Todo esse processo, da forma do uso da terra e da exploração da força de trabalho, vem se refletindo na agudização das questões sociais, deteriorizando as condições de saúde do trabalhador rural. Os que permanecem no campo sobrevivem em condições mínimas de alimentação e moradia, sem qualquer tipo de assitência médica regular ou saneamento básico, desnutridos e expoliados pelas parasitoses. Os que são excluídos desse perverso sistema de produção rural vêem na migração para os centros urbanos uma perspectiva de sobrevivência. O êxodo forçado do trabalhador rural para os grandes centros urbanos, do Nordeste ou no Sul do País, tem sido apontado como um dos fatores responsáveis pela expansão da esquistossomose. Mais uma vez cita-se o fato "da desordenada migração de pessoas infectadas pelo parasito..." sem se questionar o processo que gerou o mesmo. Lógicas como essa são geradoras de sugestões como "regulamentação da migração" como questão de segurança nacional e medida de controle (SUCEN $\left.{ }^{19}, 1982\right)$.

Embora seja inegável que a migração pode, em muitos casos, exacerbar um quadro epidemiológico, há certo risco na perspectiva de considerar o evento como uma variável que pode ser manipulada sem a apreciação das causas que geram os fluxos migratórios, e talvez sem prestar a devida atenção para os aspectos éticos e políticos da questão (Sawyer ${ }^{18}$, 1982). Os movimentos migratórios são, algumas vezes, decorrentes do desenvolvimento de modelos excludentes de industrialização, urbanização e expansão capitalista no campo (Carvalheiro ${ }^{7}, 1986$ ). Embora muitos desses movimentos tenham ocorrido na história da humanidade sem conexão causal 
com o desenvolvimento industrial, não se pode negar a relevância do modelo recente de expansão capitalista na geração de movimentos migratórios que têm importante papel epidemiológico na expansão das doenças endêmicas.

Este modelo de "desenvolvimento rural", concentrador de renda e de terras, vem causando desemprego em massa e o crescimento de uma força de trabalho pouco qualificada cujas tentativas de mobilização e reivindicações são cuidadosamente reprimidas (Singer ${ }^{21}$, 1987). Esses trabalhadores, ociosos, movimentam-se em direção das áreas urbanas atraídos pelo mercado de trabalho existente e por facilidades como assistência médica, escola, serviços e outros.

\section{URBANIZAÇÃO DA ESQUISTOSSOMOSE}

Em decorrência desta conjuntura, a esquistossomose vem se urbanizando em Pernambuco. Indivíduos infectados pelo parasito vêm tentar a sobrevivência nas cidades se inserindo no mercado produtivo (construção civil), engrossando a economia paralela (camelôs, biscateiros) ou se marginalizando totalmente do processo econômico. Passam a residir em favelas ou acampamentos temporários sem a menor condição de salubridade.

Focos peridomiciliares (ou mesmo intradomiciliares) são usualmente encontrados nas moradias de pessoas de baixa renda e, mais ainda, novos sítios de transmissão ativa da doença têm sido detectados em localidades de veraneio de classe média alta, alarmando os órgãos governamentais (Gonçalves e col. ${ }^{9}, 1992$ ).

Quadros como esse só se concretizam quando o migrante, cronicamente adoecido por determinação do processo histórico-social, encontra no espaço urbano as condições ambientais (ecológicas/biológicas) que propiciam o estabelecimento do ciclo de transmissão da esquistossomose.

\section{PERSPECTIVA DE CONTROLE}

\section{Bases Conceituais}

Uma nova concepção de saúde coletiva se faz necessária para se libertar da influência da vertente econômica que pretende fornecer explicações para as doenças a partir de indicadores exclusivamente quantitativos ou de modelos de variáveis congeladas, padronizados para todas as situações de risco.

É preciso que a investigação epidemiológica assuma a complexidade da endemia compreendendo a essência social do processo saúde/doença e a historicidade dos seus determinantes (Agudelo ${ }^{1}, 1985$ ). Essa proposta vem ganhando espaço nos meios aca- dêmicos, e alguns pesquisadores, na área de epidemiologia e controle da esquistossomose, vêm trabalhando sob essa perspectiva (Barbosa e Coimbra ${ }^{5}$, 1992; Barreto $^{6}$, 1991; Loureiro $^{13}$, 1989; Silva ${ }^{20}$, 1990; Lima $^{12}, 1994$ e Rozemberg $\left.{ }^{15}, 1994\right)$.

Operacionalizar categorias macro, tais como, processo de produção, uso e valor da terra, organização social do espaço e migração como determinantes do processo endêmico, demonstra uma capacidade explicativa maior que a dos modelos que presidem a prática da saúde coletiva $\left(\right.$ Sabroza $\left.^{16}, 1991\right)$. Mas conhecer o nível micro, específico, de determinação da esquistossomose, como padrões locais de contatos com a água e a representação que a população faz da doença, é essencial para subsidiar a elaboração de estratégias de controle em nível local, envolvendo as comunidades. Esse nível condicionante tem importante significado social, na medida em que conforma o comportamento dos indivíduos e grupos levando-os a atitudes preventivas ou práticas que resultam em maior risco de adquirir a infecção (Laurel e Gil ${ }^{11}, 1975$ ).

\section{Quadro Político}

As situações de transmissão da endemia são tão variadas como são a ecologia e os grupos sociais nos quais ela ocorre. A prevalência e intensidade de infecção, relacionadas com o destino dos dejetos, práticas agrícolas, domésticas e de lazer das comunidades afetadas pela doença, variam muito, mesmo em localidades vizinhas, sugerindo diversidade nas condições de risco e padrões de contato com a água. As medidas de controle deveriam contemplar essa diversidade, se almejam lograr efetividade nas suas ações.

A decisão política de municipalização dos serviços de saúde, com a descentralização das práticas de controle das endemias e atenção primária às populações carentes, viabiliza o conhecimento das condições específicas de risco e transmissão da doença nas localidades.

Estratégias de controle elaboradas em nível local possibilitariam o uso de categorias de análise de base epidemiológica (Possas ${ }^{14}, 1989$ ) e de abordagens etnográficas, para o melhor entendimento das especificidades.

Não se trata, no entanto, de eximir a responsabilidade do Estado na questão das endemias ou epidemias. Ações emergenciais e de combate aos vetores, quando necessárias, poderiam ser melhor racionalizadas nesse nível, e o sistema de Vigilância Epidemiológica do Estado, atuando em interação com o município, poderia normatizar e ampliar a cobertura para a detecção de casos incidentes. 


\section{Participação Popular na Esfera Municipal}

Os setores populares organizados têm demonstrado que são capazes de influenciar nas decisões da esfera local, avançando na melhoria da qualidade de vida do município (Smeke ${ }^{22}$, 1989; Valla e Stotz ${ }^{23}$, 1993). Nesse sentido, a representatividade da comunidade nas comissões municipais de saúde seria de fundamental importância, pois é nessa instância que são (ou deveriam ser) desenvolvidos os processos de elaboração, execução e avaliação das ações programáticas de controle de endemias.

\section{Participação Popular na Esfera Local}

O apoio da comunidade na elaboração e execução dos programas de controle pressupõe prática conjunta de educação em saúde, entendida enquanto processo de aquisição de conhecimento para uma ação transformadora, tanto da parte dos técnicos em saúde como dos membros da comunidade. Diversas formas de repartir o conhecimento podem ser utilizadas respeitando-se as bases culturais locais. Daí a importância da participação dos grupos sociais para a construção dos elementos culturais (representações sobre a esquistossomose), que irão subsidiar e dar coerência às estratégias de controle.

\section{CONSIDERAÇÕES FINAIS}

As proposições acima mencionadas levam a conclusão que a investigação em saúde pública necessita de um refinamento para se apropriar adequadamente da estrutura epidemiológica da doença, aliando a ética ao conhecimento técnico científico, buscando subsídios e cooperação nas diversas disciplinas para fortalecer seus instrumentos de análise.
Deste modo, a elaboração de estratégias mais coerentes para o controle das endemias trará benefícios muito mais amplos para a qualidade de vida e saúde das populações.

A comunidade científica tem o dever de ir além das denúncias, procurando atuar no sentido de provocar as necessárias transformações políticas e sociais que mudarão os rumos da saúde no País. A produção científica deve ser simultaneamente um bem cultural e um instrumento de trabalho socialmente comprometido (Barbosa ${ }^{4}$, 1987).

Em nível macro, de elaboração das políticas de saúde, grandes conquistas foram alcançadas por ocasião da VIII e IX Conferência Nacional de Saúde através da participação ativa e pressão de facções organizadas da sociedade, inclusive da comunidade científica progressista. A descentralização administrativa, quanto ao planejamento dos serviços de saúde, foi uma decisão política que torna viável, na prática, a execução das estratégias de controle acima discutidas. Cabe aos profissionais de saúde, das diversas áreas multidisciplinares, a elaboração e aplicação de modelos de intervenção locais, resgatando a proposta teórica construída politicamente nas instâncias macro deliberativas.

\section{AGRADECIMENTOS}

Aos colegas da Estação de Campo do Programa de Esquitossomose do CPqAM/FIOCRUZ pelo apoio técnico, ao Dr. Ubiracy Guida, da Fundação Nacional de Saúde de Pernambuco, pela colaboração, ao Dr. Otávio Pieri pelas informações do Município de São Lourenço da Mata, PE, e ao Dr. Wayner Vieira de Souza e Giselle Campozana, pela assessoria estatística.

\section{REFERÊNCIAS BIBLIOGRÁFICAS}

1. AGUDELO, S.F. As doenças tropicais: da análise de fatores à análise de processos. In: Nunes, E. D., org. As ciências sociais em saúde na América Latina. Brasília, OPAS, 1985. p. 474

2. ANDRADE, M.C. C. de. Lutas camponesas no Nordeste. São Paulo, Ed. Ática, 1986. (Série Princípios, 48).

3. BARBOSA, C.S. \& SILVA, C.B. Epidemiologia da esquistossomose mansônica no engenho Bela Rosa, Município de São Lourenço da Mata, PE. Cad. Saúde Pública, 8:83-7, 1992.
4. BARBOSA, F.S. Política de investigação no Brasil. Cad. Saúde Pública, 3:343-51, 1987.

5. BARBOSA, F.S. \& COIMBRA Jr., E.A.C. Alternative approaches in schistosomiasis control. Mem. Inst. Oswaldo Cruz, 87:211-20, 1992.

6. BARRETO, M. L. Geographical and socioeconomic factors relating to the distribuition of $S$. mansoni infection in an urban area of Northeastern Brazil. Bull.World Health Organ., 69:93-112, 1991. 
7. CARVALHEIRO, J.R. Processo migratório e disseminação de doenças. In: Textos de apoio de ciências sociais I. Rio de Janeiro, ENSP/ABRASCO, 1986.

8. COUTINHO, A. Medidas de controle da esquistossomose mansônica no Brasil. Rev. Assoc. Méd. do Brasil, 27:214-8, 1981.

9. GONÇALVES, J.F.; SANTANA, W.; BARBOSA, C.S.; COUTINHO, A. Esquistossomose aguda, de caráter episódico, na ilha de Itamaracá, Estado de Pernambuco. Cad. Saúde Pública, 7: 424-5, 1992.

10. HEREDIA, B.M.A. Formas de dominação e espaço social. São Paulo, Marco Zero /MCT/CNPq, 1989.

11. LAUREL, A.C.\& GIL, J.B. Morbidad, ambiente y organización social: modelo teorico para el analises de la enfermidad en el medio rural. Salud Publica Méx., 17:471-8, 1975.

12. LIMA, V.L.C. Esquistossomose no Município de Campinas, SP: processo de introdução da endemia. Fermentum: Rev. Venezolana Sociol. Antropol., 9:8-28,1994.

13. LOUREIRO, S.A. A questão do social na epidemiologia e controle da esquistossomose mansônica. Mem. Inst. Oswaldo Cruz, 84: (supl. 1) 124-31, 1989.

14. POSSAS, C. Epidemiologia e sociedade e heterogeneidade estrutural e saúde no Brasil. São Paulo, Hucitec, 1989.

15. ROZEMBERG, B. Social representation of organic process related to schistosomiasis. Cad. Saúde Pública, 10:30-46, 1994.

16. SABROZA, P.C. Espaço e produção de endemias. Rio de Janeiro, ENSP, 1991. [Trabalho apresentado ao 2ํㅡㄴ Taller da Associação Latino Americana de Medicina Social, Caracas, 1991].
17. SAMICO, M.J.A. Estudo da distribuição dos planorbídeos transmissores do S. mansoni em Piedade, Jaboatão dos Guararapes, Pe. Recife, 1989. [Monografia apresentada à Faculdade de Filosofia do Recife para obtenção do grau de Bacharel em Ciências Biológicas].

18. SAWYER, D.R. Migrações humanas e doenças tropicais: fatores sociais. In: Seminário sobre Transmissão e controle de doenças tropicais no processo de migração humana, 1981. Brasília, Centro de Documentação do Ministério da Saúde, 1982. p.121-33, 1982.

19. SECRETARIA DE ESTADO DA SAÚDE. Superintendência de Controle de Endemias. Relatório da situação da esquistossomose em São Paulo. In: Encontro sobre Esquistossomose, 5ํ, São Paulo, 1982. p. 96.

20. SILVA, L.J. Organização do espaço e doença. In: Textos de apoio à epidemiologia I. Rio de Janeiro, ABRASCO/ENSP/ FIOCRUZ, 1990. p. 283.

21. SINGER, P. Economia política da urbanização. $11^{\text {a }} \mathrm{ed}$. São Paulo, Brasilienses, 1987.

22. SMEKE, E.L.M. Saúde e democracia, experiência de gestão popular: um estudo de caso. Campinas, 1989. [Tese de Doutorado-Faculdade de Ciências Médicas da Universidade Estadual de Campinas].

23. VALLA, V.V. \& STOTZ, E. Participação popular, educação e saúde: teoria e prática. Rio de Janeiro, Relume-Dumará, 1993.

24. WORLD HEALTH ORGANIZATION. Comité de Expertos de la OMS en la Lucha contra la Esquistosomiasis, Ginebra, 1991. Informe. Ginebra, 1993. (WHO- Technical Report Series, 830). 